\title{
Relationship between After-School Physical Activity and Dietary Habits with Cardio-metabolic Risk in Low- income Children
}

\author{
Sara A. Goodrum, Timothy A. Brusseau, Janet M. Shaw, Ryan D. Burns* \\ Department of Health, Kinesiology, and Recreation, University of Utah, Salt Lake City, U.S.A \\ *Corresponding author: ryan.d.burns@utah.edu
}

\begin{abstract}
Childhood obesity is a major focus of public health. The purpose of this study was to determine whether after-school physical activity and dietary habits predict cardio-metabolic risk in a sample of ethnic minority elementary school-aged children from low-income schools. Participants were a convenience sample of 92 children $\left(3^{\text {rd }}-6^{\text {th }}\right.$ grades) recruited from four Title I schools located in a metropolitan area from the Mountain West region of the United States. Children completed portions of the After School Student Questionnaire (ASSQ) and Physical Activity Questionnaire for Children (PAQ-C) to measure after-school physical activity and nutrition, respectively. Blood pressure, waist circumference and cardio-metabolic blood markers were collected in a fasted state to calculate a continuous metabolic syndrome (MetS) composite score. Predictive relationships were analyzed using a multiple linear regression model. Neither physical activity or nutrition scores were predictive of a MetS score. However, there was a linear, positive, and moderate correlation between physical activity and nutrition scores $(r=0.29, p<0.05)$. The findings indicate that cardio-metabolic health cannot be predicted based on self-reported after-school physical activity and nutrition habits. There are many challenges that arise when analyzing cardio-metabolic health in children; however, this is an area of research that needs further analysis. A clearer understanding of these relationships can aid in identifying risk factors for disease at an earlier age and aid in future intervention development.
\end{abstract}

Keywords: children's health, metabolic syndrome, cardiovascular disease, diabetes, overweight

Cite This Article: Sara A. Goodrum, Timothy A. Brusseau, Janet M. Shaw, and Ryan D. Burns, "Relationship between After-School Physical Activity and Dietary Habits with Cardio-metabolic Risk in Low-income Children." Journal of Physical Activity Research, vol. 3, no. 1 (2018): 28-34. doi: 10.12691/jpar-3-1-5.

\section{Introduction}

The childhood obesity epidemic remains a major focus of many public health efforts in the U.S. In 2011-2012, $31.8 \%$ of children were at risk for overweight or obesity [1]. According to the Centers for Disease Control and Prevention (CDC), children that are at risk for obesity are also likely to have one or more risk factors of cardiovascular disease (CVD) such as high cholesterol and high blood pressure [2]. Freedman et al. [3] found that $70 \%$ of children with a BMI $\geq$ the $95^{\text {th }}$ percentile of the CDC growth charts had at least one and 39\% had two or more risk factors for CVD. Additionally, children classified in this category have an increased risk for developing type 2 diabetes mellitus, sleep apnea, asthma, joint and musculoskeletal discomfort, and remaining obese into adulthood [2]. The rise in childhood obesity has been directed toward the increased availability and consumption of energy dense and nutrient poor foods, decreased consumption of low-calorie and high nutrient food, increased time spent being sedentary, and decreased time spent being physically active. These factors favor a positive energy balance according to the energy balance equation, which favors weight gain $[4,5]$.

In the 2014 United States Report Card on Physical Activity for Children and Youth, overall physical activity levels were given a D-, indicating that more than half of the population did not meet the physical activity guidelines of 60 minutes or more of moderate-to-vigorous physical activity (MVPA) on at least 5 days per week [6]. According to the 2015-2020 Dietary Guidelines, presented by the United States Department of Health and Human Services (USDHHS) and the United States Department of Agriculture (USDA), children aged 8-10 do not meet the recommended intake for vegetables and dairy [7]. Additionally, approximately $16 \%$ of a child's calorie intake is from added sugar, $11 \%$ is from saturated fat, and children consume approximately 1,000 milligrams of excess sodium [7].

Cardio-metabolic risk factors approximates risk for an individual to develop heart disease or diabetes. Measurements that account for cardio-metabolic health include blood pressure, body composition, and blood cholesterol, triglyceride, and glucose levels measured when a child has been fasting [8]. When understanding the relationship between physical activity or nutrition and 
cardio-metabolic health in children, studies have identified relationships. Total physical activity is inversely associated with waist circumference, blood glucose, and triglycerides and MVPA is inversely associated with all cardio-metabolic outcomes [9]. Children who participate in higher levels of MVPA during the school day (accumulate more than 35 minutes per day) are found to have significantly lower values of waist circumference, systolic blood pressure (SBP), fasting blood glucose and triglycerides, and higher values of HDL-C in comparison to children who participate in lower levels of MVPA (18 minutes per day) [9]. Additionally, children who participate in higher levels of MVPA have more favorable metabolic health markers regardless of the amount of time spent sedentary.

For nutrition, high sodium intake can increase blood pressure and unfortunately snacks that are attractive to children are high in sodium $[8,10]$. A higher consumption of total, unsaturated, and saturated fats is associated with increased TC [11]. Fruits and vegetables have been shown to have a protective association with obesity. More than 2 servings per day of fruit or vegetables is associated with reduced blood pressure and there is further decrease in blood pressure when fruits and vegetables are combined $[8,10]$. Children who eat a high fiber breakfast have been found to have lower fasting blood glucose levels [10]. The type and amount of meat children consume can impact TC, LDL-C, HDL-C, and triglyceride levels. Lastly, high consumption of sugar-sweetened beverages leads to an increase in waist circumference, markers of inflammation, and BMI and a decrease in HDL-C [10].

When examining ways to increase children's physical activity levels and improve nutrition, the primary intervention has been through school-based interventions. School-based interventions are appealing as they reach a high a volume of students, are cost-effective, and provide benefits to children from all risk groups [12,13]. Dobbins et al. [13] conducted a systematic review to summarize the evidence of the effectiveness of promoting physical activity in schools. Positive effects were seen in increasing the duration of physical activity, increasing the participation in MVPA during school hours, and decreasing the amount of time spent watching television. For nutrition, the goal to increase fruit and vegetable consumption during lunchtime has been a focus of several interventions. These interventions focus on improving the availability, accessibility, and exposure of fruits and vegetables. A strategy to increase vegetable consumption in elementary school cafeterias by offering vegetables before other meal components increased the number of students eating vegetables [14]. A different strategy implemented in the classroom gave each child a free serving of a fruit or vegetable twice a week during a "fruit" break. This strategy led to an increase in the number of fruits and vegetable that were brought to school from home and a decrease in unhealthy snacks being brought from home [15].

Outside of school, there have been fewer interventions done to improve physical activity and nutrition. However, factors that influence children in this environment include parental habits $[11,16]$, neighborhood safety $[17,18]$, food availability [19], and socioeconomic status [13,17,18,20]. While factors have been established, understanding whether the after-school and home environment directly impacts children's cardio-metabolic health is not. With the primary focus to improve children's health coming from school-based interventions, the findings from this study could help establish a better understanding of how after-school behaviors impact health and whether or not after-school nutrition and physical activity strongly influence cardio-metabolic health in children. Additionally, these findings could assist in creating better home interventions and after-school programming by determining the key components that have a more significant impact on cardio-metabolic health as compared to others.

The primary purpose of this study was to determine the predictive relationship between after-school physical activity and dietary habits in the home environment with cardio-metabolic risk in a sample of elementary schoolaged children from low-income families. It was hypothesized that after-school nutrition and physical activity scores will be inversely related to the metabolic syndrome composite score.

Table 1. Demographic Characteristics for Participants

\begin{tabular}{|c|c|c|}
\hline Demographic & $\mathrm{n}$ & $\%$ \\
\hline \multicolumn{3}{|l|}{ Gender } \\
\hline Boys & 40 & 43.5 \\
\hline Girls & 52 & 56.5 \\
\hline Total & 92 & 100 \\
\hline \multicolumn{3}{|l|}{ Age } \\
\hline 8 & 11 & 12 \\
\hline 9 & 29 & 31.5 \\
\hline 10 & 27 & 29.3 \\
\hline 11 & 18 & 19.6 \\
\hline 12 & 7 & 7.6 \\
\hline Total & 92 & 100 \\
\hline \multicolumn{3}{|l|}{ Ethnicity } \\
\hline Caucasian & 12 & 13 \\
\hline Hispanic/Latino & 42 & 45.7 \\
\hline Asian/Pacific Islander & 12 & 13 \\
\hline American Indian/Alaskan Native & 3 & 3.3 \\
\hline Other & 20 & 21.7 \\
\hline Total & 89 & 100 \\
\hline
\end{tabular}

\section{Methods}

\subsection{Participants}

An a priori power analysis was conducted using $\mathrm{G}^{*}$ Power 3.1 [21]. Using an assumed medium sized effect $\left(\mathrm{f}^{2}=0.15\right)$, a two-sided alpha level of 0.05 , and $80 \%$ statistical power the recommended sample size was 68 participants for a multiple linear regression analysis with two predictor variables. The total recruited sample size was 120 participants. However due to incomplete data from surveys or cardio-metabolic measures, the total 
number used for analysis was 92. The participants were a convenience sample of children $\left(3^{\text {rd }}-6^{\text {th }}\right.$ grades $)$ recruited from four Title I schools located in a metropolitan area from the Mountain West region of the United States. The majority of the children that participated in this study came from low-income families. Written assent was obtained from each child and written, informed consent was obtained from one parent prior to data collection. The University Institutional Review Board approved the protocols in this study.

The demographic characteristics of the sample are presented in Table 1. Of the 92 total participants, 56.5\% were females and $43.5 \%$ were males. Over half of the participants were either 9 or 10 years of age (60.8\%) and almost half of the participants were of a Hispanic/Latino ethnic background (45.7\%). Three subjects did not indicate their ethnicity on the survey.

\subsection{Protocol}

After-school physical activity was collected using a portion of the Physical Activity Questionnaire for Older Children (PAQ-C). This questionnaire is designed for children ages 8-14 who have recess as a regular part of their school week. It is a self-administered, 7-day recall of general MVPA levels. There is evidence for this instrument to be a reliable and valid measure of general physical activity levels in children during the school year (23). For the purpose of this study, only the questions pertaining to after-school physical activity were reported. This included questions 1 and questions 5-10. For scoring, each question is worth 5 points each where 1 point represents the selection of the lowest physical activity response and 5 points represents the selection of the highest physical activity response. Question 10 was not scored in this manner as it is used to identify students who had unusual activity during the week. The mean of all the scores was then taken to find the final score where 1 indicates low physical activity and 5 indicates high physical activity. Further directions on how to obtain a composite score for each question is further described in the Physical Activity Questionnaire Manual [22].

After-school nutrition data was collected using a modified 58-item questionnaire called the After-School Student Questionnaire (ASSQ). The questionnaire examines food preferences, dietary knowledge, self-efficacy, intentions to choose healthful food options, and participation in sedentary activities and sports activities [23]. In a pilotstudy of the questionnaire, the ASSQ had acceptable internal consistency [23]. For the purpose of this study, only the nutrition behavior and food recall related sections were reported. This included questions 1-10, 18, 20-23, and question 25. Questions 5-10 were each given a score between 0 and 3. Questions 18, 20-25 were each given a score between 0 and 2. The sum of all the score was collected from the responses to be used as the total score. A higher score indicated better after-school nutrition. Scores could range from 0 to 30 .

Waist circumference was measured at the level of the superior border of the iliac crest on the participant's right side using a Gulick tape measure (Country Technology, Inc., Gays Mills, WI). All measurements were estimated to the nearest $1 \mathrm{~cm}$.
Blood pressure was measured using an electronic blood pressure device (CONTEC08A, Contec Medical Systems Co., Qinhuangdao, China). SBP and diastolic blood pressure (DBP) measurements were taken on each participant's right arm. Their right arm was rested and elevated at heart level and both feet were flat on the ground.

Each student's blood lipid profile was collected using the Cholestech LDX system (Alere Inc., Waltham, MA, USA). Individual biomarkers included TC, LDL-C, HDL-C, triglycerides, and blood glucose. A capillary blood sample was collected before the beginning of the school day between 6:30 am and 8 am. Blood samples were collected in a fasted state, which was verbally verified by the student. Blood samples were collected via a finger stick on each of the student's right index finger using a $40-\mu \mathrm{L}$ capillary tube. The blood was immediately injected into the Lipid Profile-Glucose Cassette (Alere Inc., Waltham, MA, USA) for analysis. The puncture site was then cleaned and bandaged. Disposal of all materials was placed in a biohazard container. This technique was feasible and easy to administer. Precision and accuracy of the Cholestech LDX are within the guidelines of the National Cholesterol Education Program [24].

The study commenced in April 2016 and continued for approximately 4 weeks. Children arrived to school from an overnight fast between the hours of 6:30 am and $8 \mathrm{am}$, which was verbally confirmed by the student. A blood sample was collected via a finger stick using a $40-\mu \mathrm{L}$ capillary tube. The blood was then placed in a Lipid Profile-Glucose Cassette and analyzed using the Cholestech LDX. Following the blood sample, the children had their blood pressure and waist circumference measured. Two questionnaires were then administered to the children. The AASQ was completed first and the PAQ-C was completed after the completion of the ASSQ. Children were assisted if they needed help reading or understanding questions and were given as much time as needed to complete the questionnaires.

A continuous and age- and sex-adjusted metabolic syndrome composite score (MetS) was calculated using each child's fasting triglycerides, blood glucose, HDL cholesterol, waist circumference, and SBP and DBP. An estimated mean arterial pressure (MAP) score was calculated from the SBP and DBP values using the equation: $\mathrm{MAP}=((2 \times \mathrm{DBP})+\mathrm{SBP}) / 3$. The MAP score was used for derivation of MetS as it incorporates both SBP and DBP into a single measure. Derivation of the MetS scores regressing each child's aforementioned measures onto their age and sex. Because HDL cholesterol is inversely related to cardio-metabolic risk, the HDL $\mathrm{z}$-score was multiplied by -1 . The MetS was the sum of all calculated age- and sex-adjusted z-scores. A higher MetS score will represent a more unfavorable cardio-metabolic profile. The following procedures are in accordance to those given by Eisenmann et al. [25].

\subsection{Statistical Analysis}

The design of this study was a cross-sectional and correlational. Because there was no linear relationship between the predictor variables (physical activity and nutrition scores) and the criterion variable (MetS), further 
analyses were not justified. Instead, a secondary analysis was conducted to examine the relationship between physical activity scores and nutrition scores using physical activity score as the predictor variable and nutrition score as the criterion variable. Poor food intake and physical inactivity are the two most relevant factors associated with weight gain in children $[13,26]$. The regression model of statistical assumptions was checked including linearity using a scatter plot matrix, normal distributions of residuals using a histogram, homoscedasticity using a residual versus fitted plot, and multicolinearity using leverage and tolerance statistics. Other covariates entered into the model included age and sex to control for any potentially moderating effects that were tested using interaction terms. Only predictor variables that displayed statistical significance were included in the final model. Alpha level was set a $p \leq 0.05$ and all analyses were carried out using SPSS version 23.0 statistical software package (Armonk, NY, USA).

\section{Results}

Table 2 provides the descriptive statistics for the total sample and within each sex. The nutrition scores displayed a linear, positive, moderate correlation with the physical activity scores $(r=0.29, p<0.05)$. Figure 1 is scatter plot showing the relationship between nutrition scores and physical activity scores. Nutrition scores also displayed a linear, negative, weak correlation with HDL levels $(r=-0.22, p<0.05)$.

Comparing correlations within sexes, males displayed a linear, positive, moderate correlation $(r=0.46, p<0.05)$ between nutrition scores and physical activity scores, while females did not show a significant relationship $(\mathrm{r}=0.16, p=0.24)$. Females showed a linear, negative, moderate correlation ( $\mathrm{r}=-0.33, p<0.05)$ between nutrition scores and blood glucose levels and males did not show a significant relationship $(\mathrm{r}=-0.08, p=0.62)$. Males displayed a linear, positive, moderate correlation ( $\mathrm{r}$ $=0.41, p=<0.05$ ) between waist circumference and triglyceride levels and female did not show a significant relationship $(r=0.08, p=0.59)$.

Because there was no statistical significance between the correlations of physical activity and MetS score $(\mathrm{r}=$ $0.04, \mathrm{p}=0.72$ ), and nutrition and MetS score $(\mathrm{r}=-0.02, p$ $=0.84$ ), a multiple linear regression analysis using MetS as the dependent variable and nutrition and physical activity scores as predictor variables was not conducted. However, because there was a correlation between the nutrition and physical activity scores, a secondary analysis was conducted using the nutrition score as the criterion variable and physical activity score as a predictor variable. Age and sex were included as additional predictor variables. Model 1 explained $8.1 \%$ of the variance in the nutrition score and was statistically significant ( $\mathrm{F}=7.93, p$ $=0.006)$ and model 2 explained $8.9 \%$ of the variance and was statistically significant $(\mathrm{F}=2.85, p=0.04)$. However, the change statistic for model 2 was not statistically significant indicating that model 1 provides a better model representation of the sample data. Therefore, the linear equation based on the final model parameters would be Nutrition Score $=12.23+1.28 \times$ (PA Score). The linear model indicates that a one-unit increase in PA scores is associated with 1.28-unit increase in nutrition scores.

Table 2. Descriptive Statistics (Mean and Standard Deviation)

\begin{tabular}{|c|c|c|c|}
\hline & $\begin{array}{c}\text { Total } \\
(\mathrm{N}=92)\end{array}$ & $\begin{array}{c}\text { Girls } \\
(\mathrm{n}=52)\end{array}$ & $\begin{array}{c}\text { Boys } \\
(\mathrm{n}=40)\end{array}$ \\
\hline MetS & $0.00(2.96)$ & $-0.22(2.67)$ & $0.28(3.30)$ \\
\hline $\begin{array}{c}\text { Nutrition } \\
\text { Score }\end{array}$ & $15.97(3.38)$ & $15.85(3.43)$ & $16.13(3.34)$ \\
\hline PA Score & $2.92(0.75)$ & $2.85(0.62)$ & $2.99(0.89)$ \\
\hline $\begin{array}{c}\text { HDL } \\
(\mathrm{mg} / \mathrm{dL})\end{array}$ & $48.68(12.40)$ & $46.60(11.81)$ & $51.40(12.75)$ \\
\hline \begin{tabular}{c} 
TRI (mg/dL) \\
\hline BG (mg/dL)
\end{tabular} & $105.86(55.78)$ & $107.33(57.29)$ & $103.95(54.43)$ \\
\hline \begin{tabular}{c} 
WC (cm) \\
\hline $\begin{array}{c}\text { MAP } \\
(\mathrm{mmHg})\end{array}$
\end{tabular} & $72.59(12.39)$ & $71.29(11.63)$ & $74.28(13.28)$ \\
\hline
\end{tabular}

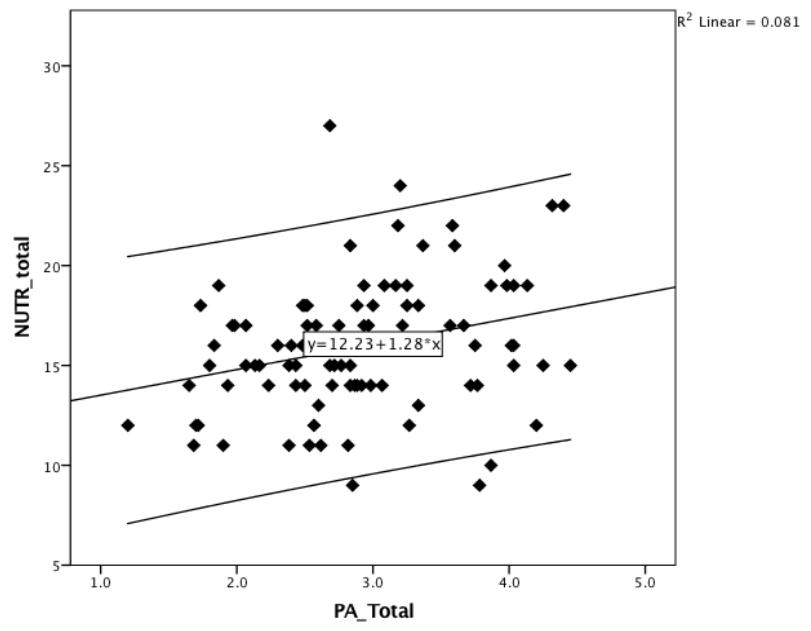

Figure 1. Scatter plot with line of best fit and 95\% CI showing the linear relationship the relationship between self-reported nutrition and physical activity scores

\section{Discussion}

The purpose of this study was to determine a predictive relationship between after-school physical activity and nutrition habits with a composite score reflective of metabolic syndrome in elementary-school aged children from low-income families. The primary finding from this study was that after-school physical activity and nutrition habits reported via survey do not predict cardio-metabolic risk in children from low-income families. A secondary analysis was conducted to characterize the relationship between after-school physical activity and after-school nutritional habits. The finding from this analysis indicated that for a 1-unit increase in the physical activity score there would be a 1.28-unit increase in the nutrition score.

Metabolic syndrome is defined as the combination of adverse cardiovascular disease and metabolic risk factors [25]. These risk factors include elevated abdominal obesity, blood pressure, fasting blood glucose, triglycerides, and lowered HDL- C [25,27]. To exhibit metabolic syndrome, an individual has 3 or more of 
the identified risk factors. For this study's population, elevated values would include greater than or equal to the $90^{\text {th }}$ percentile adjusted for age and sex for waist circumference and blood pressure, fasting glucose greater than or equal to $100 \mathrm{mg} / \mathrm{dL}$, triglycerides greater than or equal to $100 \mathrm{mg} / \mathrm{dL}$ for 8 and 9 year olds and $130 \mathrm{mg} / \mathrm{dL}$ for 10-12 year olds, and HDL-C less than or equal to 40 $\mathrm{mg} / \mathrm{dL}$ (26). There is no universal definition for metabolic syndrome in children and adolescents, but Eisenmann et al. [25] describes how they and other researchers have derived a continuous metabolic syndrome score that represents a composite cardiovascular disease/metabolic risk factor profile. This method is what was used in this study and how the composite score was determined can be found in the methods section.

There is a lack of consistent evidence showing an inverse relationship between physical activity and a continuous MetS score in children. Several factors must be considered including level of physical activity, age, sex, and form of measurement. This provides a challenge when determining a relationship. Ekelund et al. [9] reported an inverse relationship between MVPA and MetS score in 9 to 15 year olds, while DuBose et al. [28] reported MetS was not related to physical activity regardless of the intensity or meeting physical activity recommendations. Inconsistency in the literature calls for a further understanding of whether physical activity levels have a relationship to MetS. In particular, focusing on whether a particular age, sex, or physical activity level has a greater relationship. Nutrition can directly impact the risk factors that make the metabolic syndrome. For example, high sodium intake can increase blood pressure $[8,10]$. The nutrition survey utilized in this study only asked about what each child consumed the day prior to completion of the survey in the home environment. Perhaps a weeklong food long including all food consumed would have given a better idea of each child's nutrition habits.

When examining the components of the MetS and comparing them to the values listed above, $80.4 \%$ of participants had favorable HDL-C, $55 \%$ of participants between 8 and 9 years old had favorable triglyceride levels, and $80.8 \%$ of participants between 10 and 12 years old had favorable triglyceride levels. Additionally, 83.7\% had fasting blood glucose values below $100 \mathrm{mg} / \mathrm{dL}$. For blood pressure, $81.8 \%$ of 8 year olds, $70 \%$ of 9 year olds, $59.3 \%$ of 10 year olds, $77.8 \%$ of 11 year olds, and $85.7 \%$ of 12 year olds had SBP readings and for DBP, $100 \%$ of 8 year olds, $93.1 \%$ of 9 year olds, $77.8 \%$ of 10 year olds, $94.4 \%$ of 11 year olds, and $100 \%$ of 12 year olds had readings below $90^{\text {th }}$ percentile adjusted for age and sex. The percentage of children that were under the $90^{\text {th }}$ percentile for waist circumference adjusted for age and sex include 90.9, 82.8, 77.8, 94.4, and 85.7 for 8, 9, 10, 11, and 12 year olds, respectively [29]. Overall, the majority of children in this study exhibited favorable cardiometabolic health. In fact, $84.8 \%$ of the subjects had 2 or fewer of the risk factors for metabolic syndrome and $40.2 \%$ of subjects displayed none of the risk factors. With low variability, it is difficult to establish a relationship between the predictor and outcome variables.

It is important to consider that $72.8 \%$ of the children were ten years old or younger, indicating that the majority of the children were most likely pre-pubescent or in the early stages of puberty. The average age for the onset of puberty in girls occurs between 8 and 13 years of age with menarche typically occurring 2-2.5 years after the onset of puberty. The average age of menarche is between 12-12.5 years old [30]. The average age for the onset of puberty in boys occurs between 9 and 14 years of age with the mean age being 11.64 [30].

Puberty has been shown to have an effect on cardiometabolic health markers especially ones that influence the MetS [30,31,32]. One physical change that occurs during the puberty and predominantly in girls is the increase in body fat. Studies have also indicated this occurs in boys, but the results are conflicting [32]. Whether the increase in body fat is due to puberty or if there are other contributing factors such a decrease in physical activity and change in diet with aging is in question. Central accumulation of body fat can be indicated through changes in waist circumference. Body fat located in the central region of the body and estradiol levels have been shown to be related [33]. Lipid levels have also been shown to vary though each of the pubertal stages [32]. For example, TC has been shown to drop mid-puberty and then rise to adult levels towards the end. Conducting a longitudinal study that tracks change occurring throughout pubertal years would be helpful to better understand factors contributing to metabolic syndrome during this important period of development.

Analyzing cardio-metabolic health in pre-pubertal children is challenging. Several considerations need to be taken into account along with puberty including age, ethnicity, and sex [30,31,32]. Being able to identify risk for MetS early in life is important to trigger lifestyle changes that may improve health outcomes later in life. Further research is needed in tracking changes in the risk factors for metabolic syndrome in children as they transition from childhood and into adolescence.

Although not a primary aim of the study, it is important to note the relationship between physical activity and nutrition scores that was carried out in the secondary analysis. This analysis found that for a 1-unit increase in the physical activity score there would be a 1.28-unit increase in the nutrition score. Sallis et al. [34] found, in children ages 4-12, a positive association between physical activity and a healthy diet. This is important in that both components are contributors to altering cardio-metabolic health $[9,10]$. Therefore, when creating interventions to promote physical activity or healthy eating habits in children, it is important to consider that focusing on one of the components only may bring benefits to the other. An intervention that has successfully increased the intake of fruits and vegetables during lunch could lead to a secondary outcome of children increasing their levels of physical activity. This could arise from the conceptual relationship that a healthier diet leads to increased energy levels leading to an increase in physical activity. This concept should be examined in future research.

School based interventions have been effective as they reach a high volume of students in one setting [13]. Because of this, school-based interventions are the most popular forms of interventions to promote physical activity and healthy nutritional habits. However, moving forward, more research needs to be done on ways 
to implement successful interventions in the home environment or other environments outside of school as well. One way this could be done is by using interventions with a holistic approach. Interventions that originate at school have potential to translate over into the home environment. Tak et al. [15] showed this through promoting a fruit break twice a week in the classroom. After this intervention, more fruits and vegetables were brought from home as a snack during the school day. Additionally, interventions should focus on targeting children at a younger age. Programs that target children are found to be more effective than programs that target adolescents [12]. Targeting younger children could also help instill a culture where physical activity and healthy eating habits are important and a part of the norm.

There are limitations to this study that should be considered. First, the use of questionnaires created potential for bias of self-report. Self-reporting via questionnaires is beneficial in that it allows the researcher to collect from a large number of individuals and it is cost effective [35]. However, there can be drawbacks to using questionnaires especially with children. These drawbacks include the potential of the child over reporting based on what they believe is the desired answer, recall being a highly cognitive task, and easy misunderstanding of what the question may ask [35]. Although both questionnaires used in the study have shown evidence for being both reliable and valid, there is still potential for response and recall bias. For this study, the surveys were only administered once. It may have been helpful to administer the survey multiple times to account for variability that may occur in diet and physical activity on a daily and weekly basis [35]. Due to time constraints this could not be done, but should be considered for future research. Second, the convenience sample consisted of low-income children from the Mountain West region of the U.S. Because of this, the external validity of the results is questionable if generalized to other samples with different ethnic and/or socio-economic backgrounds. Finally, this study was only able to establish correlation and not causation.

Understanding how the home environment and other environments outside of school influence children's physical activity and nutritional habits in relation to cardio-metabolic health is a topic that needs further research. Future research on this topic should consider using objective measurements for physical activity and nutrition habits, as this would give a more accurate assessment [35]. If questionnaires were to be utilized again it would be important to ensure the children are adequately trained to complete the questionnaire successfully and are closely monitored. Parental aid should also be a consideration taken into account especially in food recall. Additionally, knowing whether a child is pre-pubescent, going through puberty, or post puberty is an important consideration. Hormone measurement or assessing the onset and development of puberty according to Tanner stages could be a consideration [36,37]. Puberty can influence components of MetS. It may also be more ideal to choose an age range that does not have as much variance in the potential stages of puberty that could be occurring between 8 and 12 year olds.

\section{Conclusions}

In conclusion, self-reported, after-school physical activity and nutrition did not predict a composite MetS score in children aged 8 to 12 . The inability to predict a MetS score may have risen from many factors. The need to further the understanding of how physical activity and nutrition outside of the school environment impact cardiometabolic health is of great importance. A better understanding could assist in creating more effective home-based interventions and after-school programs. Additionally, identifying how environments outside of school impact cardio-metabolic health could improve identification of health risks and diseases that children could encounter in later years of life.

\section{Acknowledgements}

The authors would like to thank the teachers, parents, and children from each school.

\section{References}

[1] Ogden CL, Carroll MD, Kit BK, Flegal KM. Prevalence of Childhood and Adult Obesity in the United States, 2011-2012. JAMA 311(8): 806-814, 2014.

[2] Centers for Disease Control and Prevention. Childhood Obesity Causes and Consequences. Atlanta, GA, 2015. http://www.cdc.gov/obesity/childhood/causes.html Accessed on January 15, 2016.

[3] Freedman DS, Mei Z, Srinivasan SR, Berenson GS, Dietz WH. Cardiovascular risk factors and excess adiposity among overweight children and adolescents: the Bogalusa Heart Study. J Pediatr 150(1): 12-17, 2007.

[4] Hall KD, Heymsfield SB, Kemnitz JW, Klein S, Schoeller DA, Speakman JR. Energy balance and its components: implications for body weight regulation. Am J Clin Nutr 95(4): 989-994, 2012.

[5] Spurrier NJ, Bell L, Wilson A, Lowe E, Golley R, Magarey AA. Minimal change in children's lifestyle behaviours and adiposity following a home-based obesity intervention: results from a pilot study. BMC Res Notes 9:26, 2016.

[6] National Physical Activity Plan. The 2014 United States report card on physical activity for children and youth. Washington, DC, 2014. http://www.physicalactivityplan.org/index.html Accessed on January 15, 2016.

[7] U.S. Department of Health and Human Services and U.S. Department of Agriculture. 2015 - 2020 Dietary Guidelines for Americans: $\quad 8^{\text {th }}$ Edition. Washington, DC, 2015. http://health.gov/dietaryguidelines/2015/guidelines/ Accessed on February 22, 2016.

[8] Kavey RW, Daniels SR, Lauer RM, Atkins DL, Hayman LL, Taubert, K. American Heart Association guidelines for primary prevention of atherosclerotic cardiovascular disease beginning in childhood. J Pediatr 142(4): 368-372, 2003.

[9] Ekelund U, Luan J, Sherar LB, Esliger DW, Griew P, Cooper A. Moderate to vigorous physical activity and sedentary time and cardio-metabolic risk factors in children and adolescents. JAMA 307(7): 704-712, 2012.

[10] Funtikova AN, Navarro E, Bawaked RA, Fito M, Schroder H. Impact of diet on cardio-metabolic health in children and adolescents. Nutr J 14: 118, 2015.

[11] Fuemmeler BF, Anderson CB, Masse LC. Parent-child relationship of directly measured physical activity. J Behav Nutr Phys Act 8:17, 2011.

[12] Birch L, Savage JS, Ventura A. Influences on the development of children's eating behaviors: from infancy to adolescence. Can J Diet Pract Res 68(1): s1-s56, 2007.

[13] Dobbins M, Husson H, DeCorby K, LaRocca RL. School-based physical activity programs for promoting physical activity and 
fitness in children and adolscents aged 6 to 18 (review). Cochrance Database Syst Rev 28(2): CD007651, 2013.

[14] Elsbernd SL, Reicks MM, Mann TL, Redden JP, Mykerezl E, Vickers ZM. Serving vegetables first: A strategy to increase vegetable consumption in elementary schools cafeterias. Appetite 96: 111-115, 2016.

[15] Tak NI, teVelde SJ, Singh AS, Brug J. The effect of a fruit and vegetable promotion intervention on unhealthy snacks during midmorning school breaks: Results on the Dutch Schoolgruiten Project. J Hum Nutr Diet 23(6): 609-615, 2010.

[16] Young EM, Fors SW, Hayes DM. Associations between perceived parent behaviors and middle school student fruit and vegetable consumption. J Nutr Educ Behav 36(1): 2-8, 2004.

[17] Dale D, Corbin CB, Dale KS. Restricting opportunities to be active during school time: do children compensate by increasing physical activity levels after school? Res Q Exerc Sport 71(3): 240-248, 2000

[18] Hume C, Salmon J, Ball K. Children's perceptions of their home and neighborhood environments, and their association with objectively measure physical activity: a qualitative and quantitative study. Health Educ Res 20(1): 1-13, 2005.

[19] Santiago-Torres M, Adams AK, Carrel AL, LaRowe TL, Schoeller DA. Home food availability, parental dietary intake, and familial eating habits influence the diet quality of urban Hispanic children. Child Obes 10(5): 408-415, 2014.

[20] Tandon PS, Zhou C, Sallis JF, Cain KL, Frank LD, Saelens BE. Home environment relationships with children's physical activity, sedentary time, and screen time by socioeconomic status. Int $\mathrm{J}$ Behav Nutr Phys Act 9:88, 2012.

[21] Faul F, Erdfelder E, Buchner A, Lang AG. Statistical power analyses using G*Power 3.1: Tests for correlation and regression analyses. Behav Res Methods 41(4): 1149-1160, 2009.

[22] Kowalski KK, Crocker PRE, Donen RM. The physical activity questionnaire for older children (PAQ-C) and adolescents (PAQ-A) manual. Canada: University of Saskatchewan, 2004.

[23] Kelder S, Hoelscher DM, Barroso CS, Walker JL, Cribb P, Hu S. The CATCH Kids Club: a pilot after-school study for improving elementary students' nutrition and physical activity. Public Health Nutr 8(2): 133-140, 2005.

[24] Issa JS, Strunz C, Giannini SD, Forti N, Diament, J. Precision and accuracy of blood lipid analyses by a portable device (Cholestech LDX). Arq Bras Cardiol 66(6): 339-342, 1996.
[25] Eisenmann JC, Laurson KR, DuBose KD, Smith BK, Donnelly JE. Construct validity of a continuous metabolic syndrome score in children. Diabetol Metab Syndr 2: 8, 2010.

[26] Skinner AC, Perrin EM, Moss LA, Skelton JA. Cardio-metabolic risks and severity of obesity in children and young adults. N Engl J Med 373(14): 1307-1317, 2015.

[27] National Heart, Lung, and Blood Institute. Expert panel on integrated guidelines for cardiovascular health and risk reduction in children and adolescents: summary report. Washington, DC, 2011. https://www.nhlbi.nih.gov/ Accessed on February 22, 2016.

[28] DuBose KD, McKune AJ, Brophy P, Geyer G, Hickner RC. The relationship between physical activity and the MetS score in children. Pediatr Exerc Sci 27(3): 364-371, 2015.

[29] Center for Disease Control and Prevention. Anthropometric reference data for children and adults: United states 2007-2010. Vital Health Stat 11(252), 2012.

[30] Wolf RM, Long D. Pubertal Development. Pediatr Rev 37(7): 292-300, 2016.

[31] Bloch CA, Clemons P, Sperling MA. Puberty decreases insulin sensitivity. J Pediatr 110(3): 481-487, 1987.

[32] Jessup A. Harrell JS. The MetS: Look for it in children and adolescents, too! Clin Diabetes 23:26-32, 2005.

[33] Davison KK, Susman EJ, Birch LL. Percent body fat at age 5 predicts earlier pubertal development among girls at age 9 . Pediatrics 111(4 Pt 1): 815-821, 2003.

[34] Sallis JF, Prochaska JJ, Taylor WC. A review of correlates of physical activity of children and adolescents. Med Sci Sports Exerc 32(5): 963-975, 2000

[35] Welk GJ, Corbin CB, Dale D. Measurement issues in the assessment of physical activity in children. Res Q Exerc Sport 71(2 Suppl): S59-S73, 2000.

[36] Marshall WA, Tanner JM. Variations in pattern of pubertal changes in girls. Arch Dis Child 44(235): 291-303, 1969.

[37] Marshall WA, Tanner JM. Variations in pattern of pubertal changes in boys. Arch Dis Child 45(239): 13-23, 1970.

[38] Ekelund U, Anderssen SA, Froberg K, Sardinha LB, Andersen LB, Brage S, European Youth Heart Study Group. Independent association of physical activity and cardiorespiratory fitness with metabolic risk factors in children: the European youth heart study. Diabetologia 50(9): 1832-1840, 2007. 